\title{
S-Bütünleyen Alt Modüller Tarafından Üretilen Öz Sınıf
}

\author{
Yilmaz DURĞUN* \\ Çukurova Üniversitesi, Adana \\ (ORCID: 0000-0002-1230-8964)
}

\begin{abstract}
$\ddot{O} \mathbf{z}$
R birimli birleşmeli bir halka olsun ve $M$ bir sağ R-modül olsun. N, M'nin bir alt modülü olsun. Eğer $Z_{2}(N)=N$ ise, $N$ ye M'nin S-bütünleyen alt modülü denir. S-bütünleyen alt modüller, tekilsiz modüller yardımıyla tanımlanan S-kapalı alt modüllerin ikilisi olarak tanımlanmıştır. Bu çalışmada, genel olarak, S-bütünleyen alt modüller yardımıyla tanımlanan S-bütünleyen kısa tam dizilerin sınıfı olan S-Büt sınıfinın bir öz sınıf olmadığı gösterilmiştir. S-Büt sınıfinı içeren en küçük öz sınıf <S-Büt> belirlenmiş ve bu öz sınıfin elemanlarının yapısı S-bütünleyen alt modüller aracılığıyla belirlenmiş̧tir. $<$ S-Büt $>$ öz sınıfinın bilinen bazı öz sınıflar ile aynı olduğu durumdaki halka yapıları belirlenmiştir. Ayrıca, değiş̧meli C-halkası üzerinde, $<$ S-Büt $>$ öz sınıfina göre projektif olan modüllerin düz modül olduğu belirlenmiştir.
\end{abstract}

Anahtar kelimeler: Bütünleyen altmodül, Öz sınıf, Goldie Burulma modülü.

\section{Proper Class Generated by S-Supplement Submodules}

\begin{abstract}
Let $\mathrm{R}$ be associative ring with unity and let $\mathrm{M}$ be a right $\mathrm{R}$-module. Let $\mathrm{N}$ be a submodule of $\mathrm{M}$. If $\mathrm{Z}_{2}(\mathrm{~N})=\mathrm{N}, \mathrm{N}$ is called S-supplement submodule of M. S-supplement submodules are defined as dual of S-closed submodules, which are defined by means of nonsingular modules. In this study, in general, it is shown that the class S-Suppl which is the class of S-supplement short exact sequences defined by the help of S-supplement submodules need not be a proper class. The smallest proper class $\langle\mathbf{S}$-Suppl $>$ containing the class $\mathbf{S}-\mathbf{S u p p l}$ is determined and the structure of the elements of $<\mathbf{S - S u p p l}>$ is determined by means of S-supplement submodules. The ring structures where the proper class $\langle\mathbf{S}-\mathbf{S u p p l}\rangle$ is the same as some known proper classes are determined. In addition, on commutative C-ring, it is determined that modules which are projective with respect to $\langle\mathbf{S}-\mathbf{S u p p l}\rangle$ are flat modules.
\end{abstract}

Keywords: Supplement submodule, Proper class, Goldie torsion module.

\section{Giriş}

Buchsbaum tarafindan, belirli bir göreceli kohomolojiye tekabül eden Ext grupları olarak hesaplanan bir kısa tam modül dizisi sınıfının hesaplanabildiği koşulları aksiyomize etmek için öz sınıflar tanımlanmıştır. Bütünleyen alt modüller tarafından belirlenen kısa tam dizilerin sınıfı Suppl ile kapalı alt modüller tarafindan belirlenen Compl sınıfının öz sınıf olduğu iyi bilinmektedir (bkz. [1, 20.7]).

$\mathrm{Bu}$ makalede ele alınan tüm halkalar birimli halkalardır. Aksi belirtilmedikçe, $\mathrm{R}$ isteğe bağlı bir halkayı belirtir ve tüm modüller sağ üniter R-modüller olacaktır. A bir R-modül olsun. $K \leq \mathbf{A}$ ile, K'nın A'nin bir alt modülü olduğu gösterilir. A'nin injektif bürümü $E(A)$ ile gösterilir. $A^{+}$ile $\operatorname{Hom}_{Z}(A, Q / Z)$ karakter modülünü belirtiriz. A bir R-modül ve $\mathrm{K} \leq \mathrm{A}$ olsun. A'nın her $\mathrm{U} \neq \mathbf{0}$ alt modülü için, $\mathrm{U} \cap \mathbf{K} \neq \mathbf{0}$ ise, K'ya A'nın bir büyük (essential) alt modülü denir ve $\mathrm{K} \leq \mathrm{e} A$ ile gösterilir. A ise K'nın genişlemesi (essential extension) olarak adlandırılır. Eğer K'nın A'da öz (proper) genişlemesi yok ise, K alt modülüne A de kapalı denir. A'nın, $\left\{\boldsymbol{m} \in \mathbf{A}: \boldsymbol{R}^{\prime} \boldsymbol{n i n}\right.$ bazı genişidealleri için $\boldsymbol{m I}=\mathbf{0}$ dır $\}$ altkümesi, A'nın tekil elemanlarının kümesidir. A’nın tekil elemanlarının kümesi Z(A) ile gösterilir ve

"Sorumlu yazar:ydurgun@cu.edu.tr

Geliş Tarihi: 18.07.2019, Kabul Tarihi: 18.10.2019 
A’nın tekil alt modülü (singular submodule) olarak adlandırılır. Tekil alt modüller burulma guruplarının genellemesidir. A modülüne, $\mathrm{Z}(\mathrm{A})=0$ ise tekilsiz (nonsingular) denir ve eğer $\mathrm{Z}(\mathrm{A})=\mathrm{A}$ ise tekil denir. R'nin sağ tekil ideali $Z_{r}(R)=Z\left(R_{R}\right)$. A modülünün ikinci tekil (veya Goldie burulma) alt modülü $Z_{2}(A)$, $\mathrm{Z}_{2}(\mathrm{~A}) / \mathrm{Z}(\mathrm{A})=\mathrm{Z}(\mathrm{A} / \mathrm{Z}(\mathrm{A}))$ eşitliği ile tanımlanır. $\mathrm{Z}_{2}(\mathrm{~A})=\mathrm{A}$ ise, $\mathrm{A}$ modülüne Goldie burulma modülü denir.

Tüm tekil modüller tarafindan üretilen burulma teorisi, Goldie burulma teorisi olarak adlandırılır. Burulma sınıfı, Goldie burulma modüllerinden ve burulmasız sınıfı ise tekilsiz modüllerden oluşmaktadır. Eğer R sağ tekilsiz ise, Goldie burulma modülleri ve tekil modüller çakışır. Goldie burulma teorisinin burulma sınıfı, homomorfik görüntüler, doğrudan toplamlar, uzantıları, alt modülleri ve injektif bürümler, burulmasız sınıfı ise alt modüller, doğrudan çarpımlar, uzantılar ve injektif bürümler altında kapalıdır. Burulma teorileri ile ilgili daha detaylı bilgi için [2,3]'e bakınız.

Kapalı alt modüller ve bütünleyen alt modüller, özellikle son 20 yılda, halka ve modül teorisi ve bağıl homolojik cebirde oynadıkları önemli rolleri nedeniyle zengin araştırma konuları oluşturmuştur. Genel olarak, bütünleyen alt modüller kapalı (veya tümleyen, tamlayan) alt modüllerin ikilisidir [1]. Bütünleyen ve tümleyen alt modüller ile ilgili olarak detaylı bir çalışma [4]'te yapılmıştır.

Kapalı alt modüller ile beraber bütünleyen alt modüllerin birçok genellemesi tanımlanmış ve incelenmiştir [5-9]. S-kapalı alt modüller, kapalı alt modüllerin bir genellemesi olarak, tekilsiz modüller yardımıyla Goodearl tarafından tanıtılmıştır [10]. S-kapalı kısa tam dizilerin sınıfı S-Compl, Compl öz sınıfının aksine bir öz sınıf değildir. S-Compl sınıfını içeren en küçük öz sınıf ve bu öz sınıfın elemanlarının yapısı [11]'te çalışılmıştır. S-kapalı alt modüller ayrıca [6]'da çalışılmıştır.

$\mathrm{Bu}$ çalışmada, S-kapalı alt modüllerin bir ikilisi olarak S-Bütünleyen alt modüller tanıtılmış ve çalışılmıştır. Genel olarak S-bütünleyen alt modüller yardımıyla tanımlanan S-bütünleyen kısa tam dizilerin sınıfı olan S-Büt bir öz sınıf olmadığını gösterdik. S-Büt sınıfını içeren en küçük öz sınıf <SBüt> belirlenmiş ve bu öz sınıfın elemanlarının yapısı S-Bütünleyen altmodülleri yardımıyla belirlenmiştir. Ayrıca bu öz sınıfın, tekilsiz modüller tarafından injektif olarak üretildiğini gösterdik. <S-Büt> öz sınıfının bilinen bazı öz sınıflar ile aynı olduğu durumdaki halka yapıları belirlenmiştir. Ayrıca değişmeli C-halkası üzerinde $<$ S-Büt $>$ öz sınıfına göre projektif olan modüllerin düz modül olduğunu gösterik.

\section{2. Öz Sınıflar}

Bu bölüm boyunca, $\mathbf{P}$, modüllerin ve modül homomorfizmlerinin kısa tam dizilerinin bir sınıfı olsun. $\mathbf{E}: 0 \rightarrow \mathrm{A} \rightarrow{ }^{\mathrm{f}} \mathrm{B} \rightarrow^{\mathrm{g}} \mathrm{C} \rightarrow 0$ kısa tam dizisi P'ye ait ise, f'nin $\mathbf{P}$-monomorfizmi olduğu ve g'nin $\mathbf{P}$ epimorfizmi olduğu söylenir. Her $\mathbf{E}$ kısa tam dizisi, izomorfizmaya bağlı olarak, bir f monomorfizması ve g epimorfizması ile tek türlü belirtilir.

Tanım: P sınıfı, aşağıdaki koşulları yerine getiriyorsa (Buchsbaum anlamında) öz sınıf olduğu söylenir (bkz., [1, 12-14]):

P-1) E'nin P'de kısa bir tam dizi olması durumunda, P E'ye izomorfik olan her bir kısa tam diziyi içerir.

P-2) P tüm parçalanan kısa tam dizileri içerir.

P-3) İki P-monomorfizmi (sırasıyla P-epimorfizmi) bileşimi, eğer bu bileşim tanımlıysa, bir Pmonomorfizmidir (sırasıly P-epimorfizmi).

P-4) Eğer g ve f monomorfizm ise ve gf bir $\mathbf{P}$-monomorfizmi ise, $\mathrm{f}$ bir $\mathbf{P}$-monomorfizmasıdır. $g$ ve $\mathrm{f}$ epimorfizmlerse ve gf bir $\mathbf{P}$-epimorfizm ise, g bir $\mathbf{P}$-epimorfizmdir.

Tüm parçalanan kısa tam dizilerinin sınıfı Split en küçük öz sınıftır ve modüllerin tüm kısa tam dizilerinin sınıfı Abs en büyük öz sınıftır. Bir diğer önemli örnek, tüm pür kısa tam dizilerin Pür öz sınıfıdır [12]. Bir $\mathbf{E}$ kısa tam dizisine pür denir eğer, her sol R-modül $\mathbf{M}$ için, $\mathbf{E} \otimes \mathbf{M}$ tamdır.

$\mathbf{P}$ sınıfını içeren tüm uygun sınıfların kesişimi, açıkça $\langle\mathbf{P}\rangle$ ile belirtilen öz sınıftır. $\langle\mathbf{P}\rangle$ sınıfı, $\mathbf{P}$ tarafından üretilen öz sınıf olarak adlandırılan, $\mathbf{P}$ yi içeren en küçük öz sınıftır. Bir $\mathbf{M}$ modülü, $\mathbf{P}$ sınıfındaki her kısa tam diziye göre projektif (sırasıyla injektif) ise, M modülü P-projektif (sırasıyla $\mathbf{P}$ injektif) olarak adlandırılır, yani $\mathbf{P}$ deki her $\mathbf{E}$ dizisi için $\operatorname{Hom}(\mathbf{M}, \mathbf{E})$ (sırasıyla $\operatorname{Hom}(\mathbf{E}, \mathbf{M})$ ) tamdır. $\langle\mathbf{P}\rangle$ 
öz sınıfı ile $\mathbf{P}$ sınıfı aynı projektif (injektif) modüllere sahip olduğuna dikkat ediniz (bkz. [4]). Bir M modülü ile başlayan (sırasıyla biten) her kısa tam dizisi $\mathbf{P}$ öz sınıfına ait ise, $\mathbf{M}$ modülüne $\mathbf{P}$-eşinjektif (sırasıyla P-eşprojektif) denir. M modüllerin verilen bir sınıfı olmak üzere, her $M \in \mathbf{M}$ nin $\overline{\boldsymbol{k}}(\mathbf{M})$ eşinjektif olduğu en küçük öz sınıf $\overline{\boldsymbol{k}}(\mathbf{M})$ ile gösterilir, ve bu öz sınıfa $\mathbf{M}$ sınıfı tarafindan eş injektif olarak üretilen öz sınıf olarak adlandırılır. $\mathbf{M}$ modüllerin verilen bir sınıfı olmak üzere, her $\mathbf{M} \in \mathbf{M}$ 'nin $\mathbf{P}$ injektif olduğu en büyük $\mathbf{P}$ öz sınıfı, $\mathbf{M}$ tarafindan injektif olarak üretilen öz sınıf olarak adlandırılır. Verilen bir $\mathrm{f}: \mathrm{A} \rightarrow \mathrm{B}$ homomorfizması ve $\mathrm{C}$ modülü için, indirgenmiş homomorfizmalar $\operatorname{Ext}^{\mathrm{R}}{ }_{1}\left(1_{C} ; \mathrm{f}\right)$ : $\operatorname{Ext}^{\mathrm{R}}{ }_{1}(\mathrm{C}, \mathrm{A}) \rightarrow \operatorname{Ext}_{1}{ }^{\mathrm{R}}(\mathrm{C}, \mathrm{B})$ ve $\operatorname{Ext}^{\mathrm{R}}{ }_{1}\left(\mathrm{f} ; 1_{\mathrm{C}}\right): \operatorname{Ext}^{\mathrm{R}}{ }_{1}(\mathrm{~B}, \mathrm{C}) \rightarrow \operatorname{Ext}^{\mathrm{R}}{ }_{1}(\mathrm{~A}, \mathrm{C})$, sirasiyla f* ve $\mathrm{f}^{*}$ ile gösterilecektir. Makale boyunca dizi ile kastedilen, modül ve modül homomorfizmalarının kısa tam dizisidir. Öz sınıflarla ilgili daha fazla bilgi için [1] ve [12]'ye bakınız.

\section{S-Bütünleyen Alt Modüller}

$\mathrm{Bu}$ bölümde S-kapalı alt modüllerin bir ikilisi olarak S-bütünleyen alt modüller tanıtılmış ve calış1lmıştır.

Tanım. A bir modül ve $\mathrm{K} \leq \mathrm{A}$ olsun. Eğer $\mathrm{Z}_{2}(\mathrm{~K})=\mathrm{K}$ ise, $\mathrm{K}$ ya A'nın $\mathrm{S}$-bütünleyen alt modülü denir. $\mathrm{S}$ bütünleyen alt modüller yardımıyla tanımlanan S-bütünleyen kısa tam dizilerin sınıfını, S-Büt ile gösterceğiz.

Genel olarak, S-Büt sınıfı bir öz sınıf değildir.

Örnek.1. $M$ Goldie burulma modülü olmasın, yani $Z_{2}(M) \neq M$ olsun. $0 \rightarrow M \rightarrow M \rightarrow 0$ kısa dizisi parçalanandır, ve $\mathrm{P}-2$ ) koşulundan dolayı her öz sınıfın içinde yer alır. Fakat $\mathrm{Z}_{2}(\mathrm{M}) \neq \mathrm{M}$ olduğundan, bu kısa tam dizi S-bütünleyen tam dizisi değildir. Dolayısıyla, S-Büt bir öz sınıf değildir.

S-Büt sınıfı, P-2) koşulu hariç bütün öz sınıf koşullarını sağladığı kolaylıkla gösterilebilir. Aşağıdaki sonuçta, S-Büt sınıfının öz sınıf olduğu halkalar karakterize edilmiştir.

Teorem.2. Aşağıdaki koşullar bir R halkası için denktir.

1. S-Büt bir öz sinıftır.

2. Her sağ R-modül bir Goldie Burulma modülüdür.

3. S-Büt=Abs

4. R sağ Goldie Burulma halkasıdır.

İspat. (1) $\rightarrow(2)$ Her sağ R-modül $\mathrm{M}$ için, $0 \rightarrow \mathrm{M} \rightarrow \mathrm{M} \rightarrow 0$ kısa dizisi parçalanandır. Kabülümüzden, bu dizi S-bütünleyen dizidir. O zaman M modülünün Goldie burulma modülü olduğunu bulmuş oluruz. $(2) \rightarrow(3)$, (3) $\rightarrow$ (1) ve (2) $\rightarrow$ (4) durumları açıktır. (4) $\rightarrow$ (2) Her sağ-R-modül bir serbest sağ-R-modülün epimorfik görüntüsüne izomorftur. Kabülümüzden R sağ Goldie burulma modül olduğundan ve Goldie burulma modüller direkt toplam altında kapalı olduklarından, her serbest sağ R-modül Goldie burulma modüldür. Goldie burulma modüllerin epimorfik görüntüsüde Goldie burulma olduğundan, her sağ Rmodülün Goldie burulma olduğu bulunur.

Kepka [14]'da yeni öz sınıfları incelemek için gerekli olan bir monomorfizm sınıfı üzerine belirli koşullar getirmiştir. Bir abel kategorisinde, herhangi bir monomorfizm, bir epimorfizmi ve kısa bir kesin diziyi oluşturduğundan, bu koşullar bu dizilerin yeni öz sınıf tiplerinin bulunmasına yol açar. Bu çalışma, genişletilmiş S-bütünleyen alt modüllerini tanımlamak için bizi motive ediyor.

Tanım. A bir modül ve $\mathrm{K} \leq \mathrm{A}$ olsun. Eğer $\mathrm{S}+\mathrm{K}=\mathrm{A}$ ve $\mathrm{S} \cap \mathrm{K}$ Goldie burulma modülü olacak şekilde bir $\mathrm{S} \leq \mathrm{A}$ var ise, $\mathrm{K}$ ya A'nın genişletilmiş $\mathrm{S}$-bütünleyen alt modül denir.

S-bütünleyen alt modüllerin genişletilmiş S-bütnleyen alt modül oldukları açıktır. Genişletilmiş S-bütünleyen kısa tam dizilerin sınıfını $\overline{\mathbf{S}-\mathbf{B u ̈ t}}$ ile göstereceğiz. $\overline{\mathbf{S}-\text { Büt }}$ sınıfının bir öz sınıf olduğu

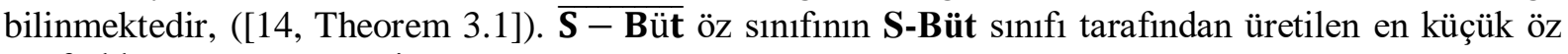
sinıf olduğunu göstereceğiz.

Teorem.3. $<$ S-Büt $>=\overline{\text { S }- \text { Büt }}$. 
İspat. $<$ S-Büt $>$ öz sınıfı S-Büt sınıfını içeren en küçük öz sınıftır. S-Büt $\overline{\subseteq S-B u ̈ t}$ olduğundan, $<$ SBüt $>\subseteq \overline{\mathbf{S}-\mathbf{B u ̈ t}}$ olduğu kolayca elde edilir. Tersine, $\mathbf{E}: 0 \rightarrow \mathrm{A} \rightarrow{ }^{\mathrm{f}} \mathrm{B} \rightarrow{ }^{\mathrm{g}} \mathrm{C} \rightarrow 0$ dizisi bir genişletilmiş $\mathrm{S}-$ bütünleyen olsun. Genellemeyi bozmadan, f homomorfizmasının bir içerme homomorfizması olduğu kabul edilebilir. O zaman $\mathrm{S}+\mathrm{A}=\mathrm{B}$ ve $\mathrm{S} \cap \mathrm{A}$ Goldie burulma modülü olacak şekilde bir $\mathrm{S} \leq \mathrm{B}$ vardır. Aşağıdaki değişmeli diyagramı düşünelim:

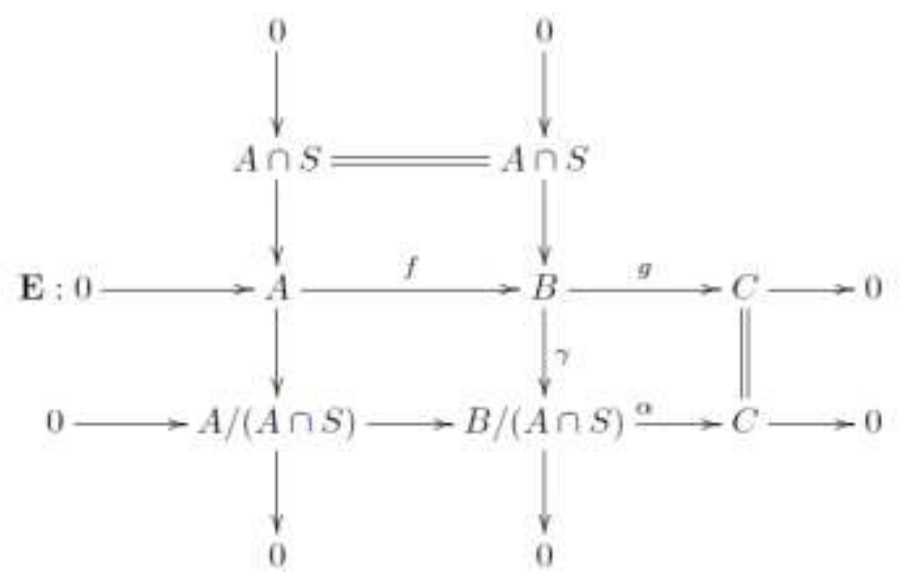

$\alpha$ bir Split-epimorfizmasıdır. Dolayısıyla, öz sınıf özelliklerinden, $\alpha$ bir $<\mathbf{S}$-Büt $>$-epimorfizması dır.

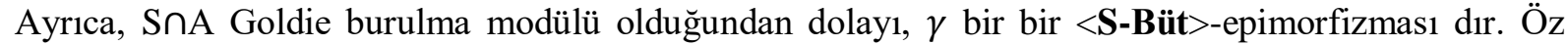
sinıfların P-3) özelliğinden, $\mathrm{g}=\alpha \gamma$ epimorfizmasının bir $\langle\mathbf{S}$-Büt $>$-epimorfizması olduğu bulunur. Buradan, $\mathbf{E} \in<$ S-Büt $>$ olduğu elde edilir.

Bir sonraki sonuçta $\overline{\mathbf{S}-\mathbf{B u ̈ t}}$ öz sınıfinın tekilsiz modüller tarafindan injektif olarak üretildiğini göstereceğiz.

Teorem.4. E: $0 \rightarrow \mathrm{A} \rightarrow{ }^{\mathrm{f}} \mathrm{B} \rightarrow{ }^{\mathrm{g}} \mathrm{B} / \mathrm{A} \rightarrow 0$ dizisinin genişletilmiş S-bütünleyen dizisi olması için gerek ve yeter koşul her $\mathrm{N}$ tekilsiz modülü için $\operatorname{Hom}(\mathbf{E}, \mathrm{N})$ nin tam olmasıdır.

İspat. $(\Rightarrow) \mathbf{E}: 0 \rightarrow \mathrm{A} \rightarrow{ }^{\mathrm{f}} \mathrm{B} \rightarrow{ }^{\mathrm{g}} \mathrm{C} \rightarrow 0$ dizisi genişletilmiş S-bütünleyen dizisi ve $\mathrm{N}$ tekilsiz modül olmak üzere $\mathrm{f}: \mathrm{A} \rightarrow \mathrm{N}$ bir homomorfizma olsun. Öz sinıf özelliklerinden, $\mathrm{f} *(\mathbf{E}): 0 \rightarrow \mathrm{N} \rightarrow{ }^{l} \mathrm{X} \rightarrow^{\alpha} \mathrm{B} / \mathrm{A} \rightarrow 0$ dizisi genişletilmiş $S$-bütünleyen dizisidir. Öyleyse, $S+N=X$ ve $S \cap N$ Goldie burulma modülü olacak şekilde bir $S \leq X$ vardır. Fakat $S \cap N$, N'nin alt modülüdür ve dolayısıyla tekilsiz modüldür. Bu yüzden $S \cap N=0$ olmak zorundadır. Buradan, $\mathrm{S} \oplus \mathrm{N}=\mathrm{X}$ olduğu bulunur. Aşağıdaki değişmeli diagramı düşünelim:

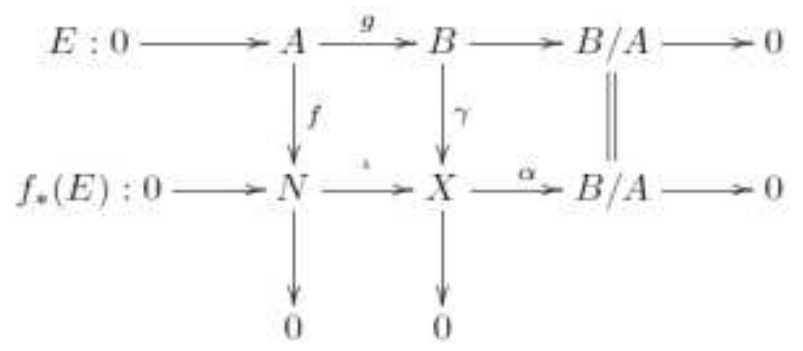

$\mathrm{f} *(\mathbf{E})$ parçalanan kısa dizi olduğundan dolayı, $\iota^{-1}=1_{\mathrm{N}}$ olacak şekilde $\iota^{-1}: \mathrm{X} \rightarrow \mathrm{N}$ homomorfizması vardır. Dolayısıyla $\left(\mathrm{l}^{-1} \gamma\right) \mathrm{g}=$ f olacak şekilde $\mathrm{I}^{-1} \gamma: \mathrm{B} \rightarrow \mathrm{N}$ homomorfizması bulmuş olduk.

$(\Leftarrow)$ Aşağıdaki diagramı düşünelim: 


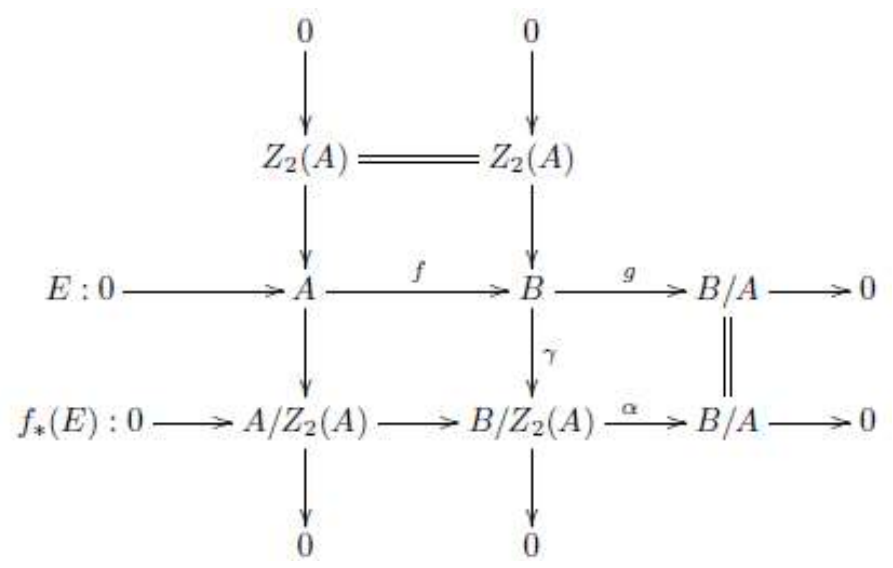

Kabülümüzden, $\mathrm{A} / \mathrm{Z}_{2}(\mathrm{~A})$ tekilsiz olduğundan dolayı, $\mathrm{f} *(\mathbf{E})$ parçalanan kısa dizidir. Dolayısıyla $\mathrm{Z}_{2}(\mathrm{~A}) \subseteq \mathrm{X}$ ve $\left(\mathrm{A} / \mathrm{Z}_{2}(\mathrm{~A})\right) \oplus\left(\mathrm{X} / \mathrm{Z}_{2}(\mathrm{~A})\right)=\mathrm{B} / \mathrm{Z}_{2}(\mathrm{~A})$ olacak şekilde bir $\mathrm{X} \subseteq \mathrm{B}$ vardır. $\mathrm{O}$ zaman $\mathrm{A}+\mathrm{X}=\mathrm{B}$ ve $\mathrm{A} \cap \mathrm{X}=\mathrm{Z}_{2}(\mathrm{~A})$ olduğu kolayca elde edilir. Buradan, $\mathbf{E}$ kısa tam dizisinin genişletilmiş S-bütünleyen olduğu elde edilir.

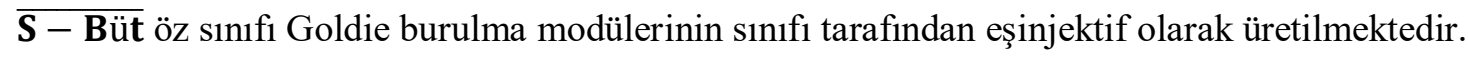

Teorem.5. T, Goldie burulma modüllerinin sınıfı olsun. $\bar{k}(\boldsymbol{T})=\overline{\mathbf{S}-\mathbf{B u ̈ t}}$.

İspat. Her Goldie Burulma modülü $\overline{\mathbf{S}-\mathbf{B u ̈ t}}$-eșinjektif olduğundan dolayı, $\bar{k}(\boldsymbol{T}) \subseteq \overline{\mathbf{S}-\mathbf{B u ̈ t}}$ olduğu açıktır. Tersi için $\mathbf{E}: 0 \rightarrow \mathrm{A} \rightarrow{ }^{\mathrm{f}} \mathrm{B} \rightarrow^{\mathrm{g}} \mathrm{C} \rightarrow 0$ dizisi genişletilmiş S-bütünleyen dizisi olsun. Öyleyse, $\mathrm{S}+\mathrm{A}=\mathrm{B}$ ve $\mathrm{S} \cap \mathrm{A}$ Goldie burulma modülü olacak şekilde bir $\mathrm{S} \leq \mathrm{B}$ vardır. Teorem 3'ün ispatında verilen

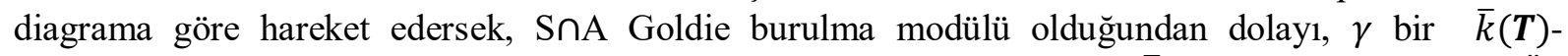
epimorfizmasıdır. $\alpha$ Split-epimorfizma olduğundan dolayı, $\alpha$ da bir $\bar{k}(\boldsymbol{T})$-epimorfizmasıdır. Öz sinıfların P-3) özelliğinden g= $\alpha \gamma$ bir $\bar{k}(\boldsymbol{T})$-epimorfizması olduğu bulunur. O zaman, $\overline{\mathbf{S}-\mathbf{B u ̈ t}} \subseteq \bar{k}(\boldsymbol{T})$ olduğu bulunur.

Her tekil sağ R-modülün injektif olduğu R halkasına sağ SI-halkası denir. R bir sağ SI-halkasıdır ancak ve ancak R sağ tekilsiz halka ve her tekil sağ R-modül yarıbasittir [10]. SI halkasının yeni bir karakterizasyonunu $\overline{\mathbf{S}-\mathbf{B u ̈ t}}$ öz sınıfı yardımıyla aşağıdaki gibi verdik.

Sonuç.6. Aşağıdaki koşullar bir R halkası için denktir.

1. Her Goldie burulma sağ R-modülü injektiftir.

2. $\mathrm{R}$ sağ SI-halkasıdır.

3. $\overline{\mathbf{S}-\mathrm{Büt}}=$ Split.

İspat. (1) $\leftrightarrow$ (2) açıktır. (1) $\rightarrow(3)$ Teorem 5 'ten elde edilir. (3) $\rightarrow(1)$ T bir Goldie burulma modülü olsun. $\mathbf{E}: 0 \rightarrow \mathrm{T} \rightarrow{ }^{\mathrm{f}} \mathrm{E}(\mathrm{T}) \rightarrow^{\mathrm{g}} \mathrm{C} \rightarrow 0$ kısa tam dizisini düşünelim. $\mathrm{T}$, Goldie burulma modülü olduğundan dolayı, $\mathbf{E}$ dizisi genişletilmiş S-bütünleyen dizisidir. O zaman, kabülümüzden, $\mathbf{E}$ parçalanandır, ve bu yüzden $\mathrm{T}$, injektif $\mathrm{E}(\mathrm{T})$ modülünün direkt toplam terimine izomorftur. Buradan T'nin injektif olduğu elde edilir.

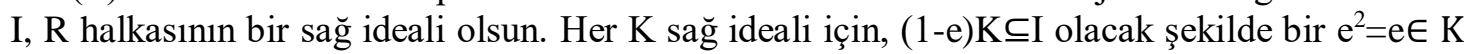
var ise, $R$ halkasına I-yarımükemmel (I-semiperfect) halka denir, [15]. $R$ sağ $Z_{2}\left(R_{R}\right)$-yarımükemmel halkadır ancak ve ancak $R$ yarımükemmel halkadır ve $J(R)=Z\left(R_{R}\right)$ dir, [16, Corollary 37].

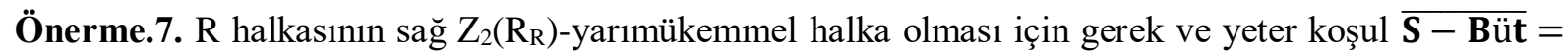
Abs olmasidir.

İspat. $(\Rightarrow) R$ nin sağ $Z_{2}\left(R_{R}\right)$-yarımükemmel halka olduğunu kabul edelim. $\mathrm{O}$ zaman, [16, Theorem 49]'dan, her tekilsiz sağ R-modül injektiftir. Teorem 4 aracılığıla $\overline{\mathbf{S}-\mathbf{B u ̈ t}}=\mathbf{A b s}$ olduğu elde edilir. $(\Leftarrow) \overline{\mathbf{S}-\mathbf{B u ̈ t}}=\mathbf{A b s}$ olduğunu kabul edelim. O zaman, Teorem 4'ten, her tekilsiz sağ R-modülün injektif olduğu elde edilir. Buradan [16, Theorem 49] yardımıyla, $R$ nin sağ $Z_{2}\left(R_{R}\right)$-yarımükemmel halka olduğu bulunur. 
Özel olarak Önerme 7'den, $R$ sağ $Z_{2}\left(R_{R}\right)$-yarımükemmel halka ise, her $\mathbf{S}$-Büt-projektif modülün projektif olduğu bulunur. Çalışmamızın son kısmında, değişmeli C-halkaları üzerinde, her S-Bütprojektif modülün düz (flat) modül olduğunu göstereceğiz.

Sıfırdan farklı bir M modülünün 0 ve $M$ den başka alt modülü yoksa, $M$ ye basit modül denir.

Önerme.8. Değişmeli R halkası üzerinde her injektif olmayan basit modül tekildir.

İspat. U injektif olmayan basit bir modül olsun. U'nun projektif bir modül olduğunu varsayalım. $\mathrm{O}$ zaman $\mathrm{U}^{++}$injektif olur, çünkü $\mathrm{U \cong} \mathrm{U}^{+}$ve bir projektif (düz) modülün karakter modülü injektiftir. $\mathrm{U}$, [17, Proposition 5.3.9]'dan, $\mathrm{U}^{++}$injektif modülünün saf alt modülüdür. Fakat U Saf-injektif modüldür, dolayısıyla $U$ injektif $\mathrm{U}^{++}$modülünün doğrudan toplam terimine izomorftur. Buradan U'nin injektif olduğu elde edilir, çelişki. Dolayısıyla, U tekildir.

$\mathrm{R}$ halkasının her büyük temel sağ I ideali için $\operatorname{Soc}(\mathrm{R} / \mathrm{I}) \neq 0$ ise, yani $\mathrm{R} / \mathrm{I}$ basit alt modüle sahip ise, R'nin sağ C-halkası olduğu söylenir, $[1,10.10]$. Sol mükemmel halkalar ve sağ yarı-artin halkaları sağ C-halkalarının iyi bilinen örnekleridir. Sağ C-halkalarının iyi bilinen karakterizasonu şu şekildedir: $\mathrm{R}$, her basit $\mathrm{S}$ sağ $\mathrm{R}$-modülü için $\mathrm{Ext}_{1}{ }^{\mathrm{R}}(\mathrm{S}, \mathrm{M})=0$ iken M'nin injektif olduğunu ima etmesi durumunda sağ C-halkasıdır [18, Lemma 4]. Son olarak, değişmeli C-halkası üzerinde, her S-Büt-projektif modülün

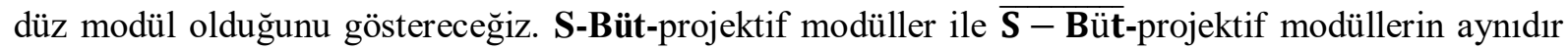
[4].

Teorem. 9. R, değişmeli C-halkası olsun. Her S-Büt-projektif modül düz modüldür.

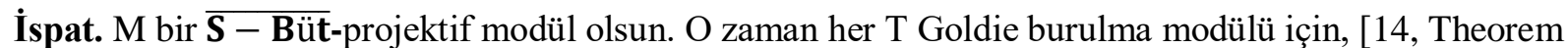
3.1]'den, $\operatorname{Ext}_{1}{ }^{R}(M, T)=0$ olur. Özel olarak, Önerme 8'den, her $S$ basit modülü için $\operatorname{Ext}_{1}{ }^{R}(M, S)=0$ olur. Eğer $\mathrm{R}$ değişmeli halka ve $\mathrm{E}$ bir injektif eş-üreteç(cogenerator) ise, $\operatorname{Hom}(\mathrm{S}, \mathrm{E}) \cong \mathbf{S}$ olduğunu hatırlatalım. O zaman, her basit $\mathrm{S}$ modülü için, $\operatorname{Ext}_{1}{ }^{\mathrm{R}}(\mathrm{M}, \operatorname{Hom}(\mathrm{S}, \mathrm{Q} / \mathrm{Z}))=0$ olur. Standart birinci ve ikinci eşdeğer(adjoint) izomorfizm teoremlerinden $\operatorname{Ext}_{1}{ }^{\mathrm{R}}(\mathrm{S}, \operatorname{Hom}(\mathrm{M}, \mathrm{Q} / \mathrm{Z})) \cong \operatorname{Hom}\left(\operatorname{Tor}^{1}(\mathrm{M}, \mathrm{S}), \mathrm{Q} / \mathrm{Z}\right) \cong$ $\operatorname{Ext}_{1}{ }^{\mathrm{R}}(\mathrm{M}, \operatorname{Hom}(\mathrm{S}, \mathrm{Q} / \mathrm{Z}))=0$, [19, Teorem 12.75]. R bir C-halkası olduğundan, Hom(M, Q/Z) injektif modüldür. Dolayısıyla, [18, Proposition 3.54] yardımıyla, M düz modüldür.

\section{Teşekkür}

$\mathrm{Bu}$ araştırma, Çukurova Üniversitesi Bilimsel Araştırma Fonu tarafından desteklenmiştir (Proje Numaras1: 10871).

\section{Yazarların Katkısı}

Bu makalenin hazırlanması için gerekli çalışmaların tümü yazar tarafından yapılmıştır.

\section{Çıkar Çatışması Beyanı}

Yazarlar arasında herhangi bir çıkar çatışması bulunmamaktadır.

\section{Araştırma ve Yayın Etiği Beyanı}

Yapılan çalışmada araştırma ve yayın etiğine uyulmuştur.

\section{Kaynaklar}

[1] Clark J., Lomp C., Vanaja N., Wisbauer R. 206. Lifting modules. Birkhauser Verlag, Basel.

[2] Crivei S. 2004. Injective modules relative to torsion theories. EFES Publishing House, ClujNapoca.

[3] Golan J.S. 1986. Torsion Theories. Longman Scientific \&Technical, Harlow. 
[4] Alizade R., Mermut E. 2015. Proper classes related with complements and supplements. Palestine Journal of Mathematics, 4 (Spec. 1): 471-489.

[5] Pancar A., Türkmen B.N., Nebiyev C., Türkmen E. 2019. On a new variation of injective modules. Communications, Series A1: Mathematics and Statistics, 68 (1): 702-711.

[6] Kara Y., Tercan A. 2018. When some complement of a z-closed submodule is a summand. Communications in Algebra, 46 (7): 3071-3078.

[7] Tütüncü D.K., Toksoy S.E. 2013. Absolute co-supplement and absolute co-coclosed modules. Hacettepe Journal of Mathematics and Statistics, 42 (1): 67-79.

[8] Sözen E. Ö., Eren Ş. 2017. Modules that Have a $\delta$-Supplement in Every Extension. European Journal of Pure and Applied Mathematics, 10 (4): 730-738.

[9] Koşar B., Türkmen B.N. 2016. A generalization of oplus-cofinitely supplemented modules. Bulletin of the Iranian Mathematical Society, 42 (1): 91-99.

[10] Goodearl K.R. 1972. Singular torsion and the splitting properties. American Mathematical Society, Providence, R.I.

[11] Durğun Y., Ozdemir S. 2017. On S-closed submodules. Journal of the Korean Mathematical Society, 54 (4): 1281-1299.

[12] Sklyarenko E.G. 1978. Relative homological algebra in the category of modules. Uspekhi Matematicheskikh Nauk, 33 (3): 85-120.

[13] Pancar A. 1997. Generation of proper classes of short exact sequences. International Journal of Mathematics and Mathematical Sciences, 20 (3): 465-473.

[14] Kepka T. 1973. On one class of purities. Commentationes Mathematicae Universitatis Carolinae, 14: 139-154.

[15] Yousif M.F., Zhou Y. 2002. Semiregular, semiperfect and perfect rings relative to an ideal. Rocky Mountain Journal of Mathematics, 32: 1651-1671.

[16] Nicholson W.K., Zhou Y. 2005. Strong Lifting. Journal of Algebra. 285: 795-818.

[17] Enochs E., Jenda O.M.G. 2000. Relative homological algebra. de Gruyter Expositions in Mathematics, de Gruyter, Berlin.

[18] Smith P.F. 1981. Injective modules and prime ideals. Communications in Algebra, 9 (9): 989999.

[19] Rotman J. 1979. An introduction to homological algebra. Academic Press, New York. 